Pacific Journal of Mathematics

OPERATORS SATISFYING A $G_{1}$ CONDITION 


\title{
OPERATORS SATISFYING A $G_{1}$ CONDITION
}

\author{
C. R. Putnam
}

An operator $T$ on a Hilbert space is said to be $G_{1}$ if $\left\|(T-z)^{-1}\right\|=1 / \operatorname{dist}(z, \sigma(T))$ for $z \notin \sigma(T)$ and completely $G_{1}$ if, in addition, $T$ has no normal part. Certain results are obtained concerning the spectra of completely $G_{1}$ operators and of their real parts. It is shown in particular that there exist completely $G_{1}$ operators having spectra of zero Hausdorff dimension. Some sparseness conditions on the spectrum are given which assure that a $G_{1}$ operator has a normal part.

1. Introduction. All operators considered in this paper will be bounded (linear) on a Hilbert space $\mathfrak{S}$ of elements $x$. For any such operator $T$ it is well-known (and due to Wintner [26]) that

$$
\left\|(T-z)^{-1}\right\| \geqq 1 / \operatorname{dist}(z, \sigma(T))
$$

for $z \notin \sigma(T)$ and $\left\|(T-z)^{-1}\right\| \leqq 1 / \operatorname{dist}\left(z, W^{-}(T)\right)$ for $z \notin W^{-}(T)$, where $\sigma(T)$ denotes the spectrum of $T$ and $W^{-}(T)$ denotes the (convex) closure of the numerical range $W(T)=\{(T x, x):\|x\|=1\}$. An operator $T$ is said to be $G_{1}$ (or to satisfy a $G_{1}$ condition, or to be of class $G_{1}$ ) if

$$
\left\|(T-z)^{-1}\right\|=1 / \operatorname{dist}(z, \sigma(T)) \quad \text { for } \quad z \notin \sigma(T) .
$$

For instance, (1.1) holds for operators $T$ which are normal $\left(T^{*} T-\right.$ $\left.T T^{*}=0\right)$, more generally, for those which are subnormal ( $T$ has a normal extension on a larger Hilbert space), and still more generally, for hyponormal operators $\left(T^{*} T-T T^{*} \geqq 0\right)$. The inclusions indicated here,

$$
\text { normals } \subset \text { subnormals } \subset \text { hyponormals } \subset\left(G_{1}\right),
$$

are all proper and, needless to say, the simple stratification (1.2) can be interstitially (and endlessly) refined. In this connection, see the brief survey in Putnam [16].

An operator $T$ will be called completely $G_{1}$ if $T$ is $G_{1}$ and if, in addition, $T$ has no normal part, that is, $T$ has no reducing subspace on which it is normal. Similarly, one has corresponding definitions of completely subnormal or completely hyponormal operators. It is well-known that every compact set of the plane is the spectrum of some normal operator. Moreover, necessary and sufficient conditions are known in order that a compact set be the spectrum of a completely subnormal operator (Clancey and Putnam [4]) or of a completely 
hyponormal operator (Putnam [15], [17]). On the other hand, no such conditions are known for the class of completely $G_{1}$ operators.

It may be noted that if $T$ is $G_{1}$ and if $\sigma(T)$ is finite, in particular, if $\mathscr{H}$ is finite-dimensional, then necessarily $T$ is normal. In fact, Stampfli [20], p. 473, shows that if $T$ is $G_{1}$ and if $z_{0}$ is an isolated point of $\sigma(T)$ then $z_{0}$ is a normal eigenvalue of $T$, that is, $z_{0} \in \sigma_{p}(T)$, the point spectrum of $T$, and the corresponding eigenvectors form a reducing space of $T$ on which $T$ is normal. (For some related results, see also Hildebrandt [8], p. 234, and Luecke [10], p. 631.) More generally, it was shown by Stampfli ([22], [23]) that if $T$ is $G_{1}$ and if $\sigma(T)$ is a subset of a smooth $\left(C^{2}\right)$ curve then $T$ is normal. In fact, he even obtains a local version of this result. Thus, if $z_{0} \in \sigma(T)$ and if $D$ is an open disk centered at $z_{0}$ for which $\sigma(T) \cap D$ lies on a smooth curve and for which $T$ is only locally $G_{1}$, so that (1.1) is assumed only in $D-\sigma(T)$, then $T$ has a representation $T=T_{1} \oplus T_{2}$ where $T_{1}$ is normal with spectrum $(\sigma(T) \cap D)^{-}$and $T_{2}$ has a spectrum contained in $\sigma(T)-D$. On the other hand, as Stampfli has shown ([20], p. 474; [22], p. 9), it is possible that (1.1) holds and that $\sigma(T)$ is even a countable subset of a curve $z=z(t), 0 \leqq t \leqq 1$, where $z(t)$ is $C^{2}$ for $0 \leqq t<1$, but $T$ fails to be normal. In [10], Luecke shows that if $\sigma(T)$ is countable and has the property that for any $z \in \sigma(T)$ there exists some $w \notin \sigma(T)$ for which $|z-w|=$ $\operatorname{dist}(w, \sigma(T))$, then, in general, $T$ need not be normal. However, if, in addition, $T$ is assumed to be a scalar operator, then it must indeed be normal.

All of this suggests that a simple necessary and sufficient condition on a compact set in order that it be the spectrum of a completely $G_{1}$ operator is not easily obtained. In fact, even such a condition on a countable compact set in order that it be the spectrum of a nonnormal operator of class $G_{1}$ is not known. (A sufficient condition for normality is that of Luecke [10] mentioned above; another is given in Theorem 2 below.) Of course, any $G_{1}$ operator having a countable spectrum certainly has a normal part. It is thus clear that a necessary condition on a compact set, $X$, in order that it be the spectrum of a completely $G_{1}$ operator is that $X$ be perfect. In order to describe certain types of sets $X$ occurring below, it will be convenient to recall the definition of Hausdorff measure.

A "measure function" $h(t)$ is an increasing continuous function on $0 \leqq t<\infty$ satisfying $h(0)=0$. For a bounded set, $X$, of the complex plane and a fixed $\delta>0$ let $\Gamma=\left\{D_{1}, D_{2}, \cdots\right\}$ be any countable covering of $X$ by open disks $D_{j}$ of radius $\delta_{j} \leqq \delta$. Then $\Lambda_{h}(X)=$ $\lim _{\tilde{i} \rightarrow 0}\left[\inf \sum_{j=1}^{\infty} h\left(\delta_{j}\right)\right]$ exists and is the Hausdorff $h$-measure of $X$. (See Garnett [5], p. 58; also Carleson [2], Rogers [19].) If $h(t)=t^{r}, r>0$, then $\Lambda_{h}(X)$ is the $r$-dimensional Hausdorff measure of $X$. In par- 
ticular, a nonempty set $X$ is said to have Hausdorff dimension $=0$ if $\Lambda_{h}(X)=0$ for all $h=t^{r}, r>0$.

2. THEOREM 1. For any given measure function $h$ there exists a perfect set $X$ of the complex plane and 'a completely $G_{1}$ operator $T$ for which $X=\sigma(T)$ has Hausdorff h-measure $=0$.

It may be noted that, in particular, there exist completely $G_{1}$ operators with spectra of Hausdorff dimension $=0$. That the function $h$ of Theorem 1 be preassigned is an essential requirement however. In fact, the condition that $\Lambda_{h}(\sigma(T))=0$ for all measure functions $h$ is sufficient (as well as necessary) in order that $\sigma(T)$ be countable; see Rogers [19], p. 67.

Proof. As in Stampfli ([20], [22]), consider the matrix

$$
A=\left(\begin{array}{ll}
0 & 1 \\
0 & 0
\end{array}\right)
$$

acting on a two-dimensional Hilbert space, so that $(A-z)^{-1}=$ $\left(\begin{array}{cc}-1 / z & -1 / z^{2} \\ 0 & -1 / z\end{array}\right)$, and hence $\left\|(A-z)^{-1}\right\| \leqq 1 /|z|+1 /|z|^{2}$ for all $z \notin \sigma(A)=$ \{0\}. Note also that $W(A)\left(=W^{-}(A)\right)=\{z:|z| \leqq 1 / 2\}$ and $\|A\|=1$. Then $\|(A-z)^{-1}|| \leqq(|z|-1 / 2)^{-1}$ for $|z|>1 / 2$ and clearly there exists a countable set $\alpha=\left\{z_{1}, z_{2}, \cdots\right\} \subset\{z: 0<|z|<1\}$ satisfying $z_{n} \rightarrow 0$ as $n \rightarrow \infty$ and such that

$$
\left\|(A-z)^{-1}\right\| \leqq 1 / \operatorname{dist}(z, \alpha) \text { for } z \neq 0 .
$$

Next, choose a sequence of nonoverlapping open disks $\left\{D_{1}, D_{2}, \cdots\right\}$, where each $D_{n}$ has center $z_{n}$ and is contained in $\{z: 0<|z|<1\}$. Let $A_{n}=a_{n} A+z_{n}$, where $0<a_{n}<$ radius $D_{n}$, so that $\left\|A_{n}-z_{n}\right\|=$ radius $D_{n}$ and $\sigma\left(A_{n}\right)=\left\{z_{n}\right\}$. Then, for each $n=1,2, \cdots$, choose a countable set $\alpha_{n}=\left\{z_{n 1}, z_{n 2}, \cdots\right\} \subset D_{n}$ satisfying $z_{n k} \neq z_{n}$ and $z_{n k} \rightarrow z_{n}$ as $k \rightarrow \infty$ and the inequality $\left\|\left(A_{n}-z\right)^{-1}\right\| \leqq 1 / \operatorname{dist}\left(z, \alpha_{n}\right)$ for $z \neq z_{n}$. Thus, if $T_{0}=A$ and $T_{1}=\sum \oplus A_{n}$, one sees that

$$
\begin{aligned}
& T=T_{0} \oplus T_{1} \text { satisfies }\left\|(T-z)^{-1}\right\| \leqq 1 / \operatorname{dist}\left(z, \cup \alpha_{n}\right) \text { for } \\
& \quad z \notin \sigma(T)=\{0\} \cup \alpha .
\end{aligned}
$$

In the next step each of the disks $D_{n}$ plays the role of the containing disk $\{z:|z|<1\}$ in the previous construction. Thus, for each $n=1,2, \cdots$, one chooses a sequence of nonoverlapping open disks $\left\{D_{n 1}, D_{n 2}, \cdots\right\}$, contained in $D_{n}$ and clustering at $z_{n}$, and obtains a new operator $T_{2}$ for which $T=T_{0} \oplus T_{1} \oplus T_{2}$ satisfies a condition analogous to (2.2) for $T=T_{0}$ and to (2.3) for $T=T_{0} \oplus T_{1}$. Continu- 
ation of this process leads to an operator $T=\sum_{k=0}^{\infty} \oplus T_{k}$ satisfying

$$
\left\|(T-z)^{-1}\right\| \leqq 1 / \operatorname{dist}(z, X) \text { for } z \notin X,
$$

where $X$ is the closure of the set of all centers of circles occurring in the above construction. Since $X \subset \sigma(T)$ then, by $(2.4), \sigma(T)=X$ and $T$ satisfies (1.1). Moreover, it is clear that $T$ is a completely $G_{1}$ operator. Further, the inclusions

$$
\{z: z<1\} \supset\left[\cup D_{n} \cup\{0\}\right] \supset\left[\cup D_{n k} \cup\left\{0, z_{1}, z_{2}, \cdots\right\}\right] \supset \cdots \supset \sigma(T)
$$

show that, for any given measure function $h$, one can always choose the countable collection of disks $\left\{D_{n}\right\},\left\{D_{n k}\right\}, \cdots$, in such a way that $\sigma(T)$ has Hausdorff $h$-measure $=0$. This completes the proof of Theorem 1.

CoROLlaRY 1. If $X$ denotes an arbitrary compact set of the plane and if $h$ is any measure function, then there exists a perfect set $P \supset X$ and a completely $G_{1}$ operator $T$ such that $P-X$ has Hausdorff $h$-measure $=0$ and $\sigma(T)=P$.

Proof. Let $\left\{z_{1}, z_{2}, \cdots\right\}$ be any countable subset of $X$ dense in $X$. For each $n=1,2, \cdots$, let $D_{n}$ be an open disk centered at $z_{n}$ and suppose that diam $D_{n} \rightarrow 0$ as $n \rightarrow \infty$. Then let $T_{n}$ be a completely $G_{1}$ operator having spectrum of Hausdorff $h$-measure $=0$ and such that $z_{n} \in \sigma\left(T_{n}\right) \subset D_{n}$. One need only choose $T_{n}$, for instance, to be an appropriate linear function of the operator $T$ constructed in the proof of Theorem 1. (Note that the $G_{1}$ property is invariant under linear transformations; see Luecke [11], p. 36.) If $T=\sum \oplus T_{n}$ then, since each $T_{n}$ is $G_{1}, \sigma(T)=\left(\cup \sigma\left(T_{n}\right)\right)^{-}$and hence, since diam $D_{n} \rightarrow 0$ as $n \rightarrow$ $\infty, \sigma(T)=\bigcup \sigma\left(T_{n}\right) \cup X=P$ satisfies the conditions stated in the corollary.

A related result is the following

COROLlaRy 2. If $B$ is any operator and $h$ is any measure function there exists a completely $G_{1}$ operator $T$ for which $B \oplus T$ is also $G_{1}$ and $\sigma(T) \subset\{\partial(\sigma(B)) \cup \beta\}$ where $\beta$ has Hausdorff $h$-measure $=0$.

Proof. Choose a sequence of points $\alpha=\left\{z_{1}, z_{2}, \cdots\right\}$ in such a way that no $z_{n}$ lies in $\sigma(B), \operatorname{dist}\left(z_{n}, \sigma(T)\right) \rightarrow 0$ as $n \rightarrow \infty$, and such that $\left\|(B-z)^{-1}\right\| \leqq 1 / \operatorname{dist}(z, \alpha)$ for $z \notin \sigma(B)$. Then choose a sequence of open disks $\left\{D_{1}, D_{2}, \cdots\right\}$, where $z_{n}$ is the center of $D_{n}$, satisfying $D_{n} \cap \sigma(B)=\varnothing$ and $\operatorname{diam} D_{n} \rightarrow 0$ as $n \rightarrow \infty$, so that the $D_{n}$ 's cluster only on the set $\partial(\sigma(B))$. If $T_{1}, T_{2}, \cdots$ are $G_{1}$ operators such that $z_{n} \in \sigma\left(T_{n}\right) \subset D_{n}$ and $\sigma\left(T_{n}\right)$ has Hausdorff $h$-measure $=0$, then $T=$ 
$\Sigma \oplus T_{n}$ satisfies the conditions stated in the corollary.

3. Some lemmas. If $\left\{A_{1}, A_{2}, \cdots\right\}$ is a decreasing sequence of self-adjoint operators then the $A_{n}$ converge strongly to a (self-adjoint) operator $A$, a result due to Vigier (see Riesz and Sz.-Nagy [18], p. 263). In particular, if each $A_{n}$ is an orthogonal projection, so also is $A$. Further, it is well-known that a projection $P\left(P=P^{2}\right)$ is orthogonal if and only if $\|P\| \leqq 1$. We shall need need the following generalization to arbitrary projections $P_{n}$ of the above results.

Lemma 1. Let $\left\{P_{1}, P_{2}, \cdots\right\}$ be a sequence of projections $\left(P_{n}=P_{n}^{2}\right)$ satisfying

$$
P_{n} P_{n+p}=P_{n+p} \quad(n=1,2, \cdots ; p=0,1,2, \cdots)
$$

and

$$
\limsup _{n \rightarrow \infty}\left\|P_{n}\right\| \leqq 1
$$

Then the $P_{n}$ converge strongly as $n \rightarrow \infty$ to an orthogonal projection.

Proof. First, let $\mathrm{P}$ denote any projection and let $t \geqq 0$ satisfy

$$
\|P\| \leqq 1+t .
$$

Since $P^{2}=P$, the range of $P^{*}$ is orthogonal to the range of $I-P$ and hence, if $x$ is arbitrary in $\mathfrak{S}$ and $y=P^{*} x$, then $y=P^{*} y \perp(I-P) y$. Since $P y=y-(I-P) y$, then

$$
\|y\|^{2}+\|(I-P) y\|^{2}=\|P y\|^{2} \leqq(1+t)^{2}\|y\|^{2},
$$

and so $\left\|(I-P) P^{*} x\right\|^{2} \leqq\left(2 t+t^{2}\right)\left\|P^{*} x\right\|^{2}$. Consequently,

$$
\left\|P-P P^{*}\right\|=\left\|P^{*}-P P^{*}\right\| \leqq t^{1 / 2}(2+t)^{1 / 2}(1+t),
$$

and hence

$$
\left\|P-P^{*}\right\| \leqq 2 t^{1 / 2}(2+t)^{1 / 2}(1+t) .
$$

Relations (3.2) and (3.5) (with $P$ replaced by $P_{n}$ ) imply that $\left\|P_{n}-P_{n}^{*}\right\| \rightarrow 0$ as $n \rightarrow \infty$. Further, if $Q_{n}=P_{n} P_{n}^{*}$, also $\left\|Q_{n}-P_{n}\right\| \rightarrow$ 0 as $n \rightarrow \infty$, and hence, by (3.1), $\left\|Q_{n} Q_{n+p}-Q_{n+p}\right\| \rightarrow 0$ as $n \rightarrow \infty$ (uniformly in $p \geqq 0$ ). Similarly, $\left\|Q_{n} Q_{n+p}-Q_{n+p} Q_{n}\right\| \rightarrow 0$ as $n \rightarrow \infty$ (uniformly in $p \geqq 0$ ) and hence also $\left\|Q_{n}\left(I-Q_{n+p}\right)-Q_{n}^{1 / 2}\left(I-Q_{n+p}\right) Q_{n}^{1 / 2}\right\| \rightarrow$ 0 (uniformly in $p \geqq 0$ ). It follows that there exists a sequence of positive numbers $\left\{t_{1}, t_{2}, \cdots,\right\}$ with limit 0 for which

$$
A_{n p} \equiv Q_{n}-Q_{n+p}+t_{n} \geqq 0 \text { for all } n \geqq 1 \text { and } p \geqq 0 \text {. }
$$


If $x$ is arbitrary in $\mathscr{S}$, then clearly one can choose integers $n=$ $n_{k} \rightarrow \infty$ and $p=p_{k} \rightarrow \infty$ so that $\left(Q_{n_{k}} x, x\right) \rightarrow \lim _{\inf _{n \rightarrow \infty}}\left(Q_{n} x, x\right)$ and also $\left(Q_{n_{k}+p_{k}} x, x\right) \rightarrow \lim \sup _{n \rightarrow \infty}\left(Q_{n} x, x\right)$. Hence, by (3.6),

$$
\lim _{n \rightarrow \infty}\left(Q_{n} x, x\right) \text { exists, for each } x \text { in } \mathscr{B} \text {. }
$$

An argument like that in Riesz and Sz.-Nagy [18], p. 263, shows that $\left\|A_{n p} x\right\|^{4}=\left(A_{n p} x, A_{n p} x\right)^{2} \leqq\left(A_{n p} x, x\right)\left(A_{n p}^{2} x, A_{n p} x\right)$ and hence, by (3.7) and the definition of $A_{n p}$ in (3.6), $\left(Q_{n}-Q_{n+p}\right) x \rightarrow 0$ (strongly) as $n \rightarrow$ $\infty$ (uniformly in $p \geqq 0$ ), so that $Q=s$ - $\lim _{n \rightarrow \infty} Q_{n}$ exists and is selfadjoint. Since $\left\|Q_{n}-P_{n}\right\| \rightarrow 0$, then $s-\lim _{n \rightarrow \infty} P_{n}=Q$ is an orthogonal projection and the proof of Lemma 1 is complete.

Lemma 2. Let $T$ be a $G_{1}$ operator and suppose that $z_{0} \in \sigma(T)$. In addition, suppose that there exists a sequence of circles $C_{n}=$ $\left\{z:\left|z-z_{0}\right|=r_{n}\right\}, n=1,2, \cdots$, lying in the resolvent set of $T$, and for which $r_{1}>r_{1}>\cdots \rightarrow 0$ and

$$
r_{n} / \operatorname{dist}\left(C_{n}, \sigma(T)\right) \longrightarrow 1 \text { as } n \longrightarrow \infty \text {. }
$$

If each $C_{n}$ is positively oriented and if $P_{n}$ denotes the projection

$$
P_{n}=-(2 \pi i)^{-1} \int_{C_{n}}(T-z)^{-1} d z \quad(n=1,2, \cdots),
$$

then $P_{n} \rightarrow P$ (strongly), where $P$ is an orthogonal projection commuting with $T$, and

$$
\left(T-z_{0}\right) P=0
$$

Proof. That the $P_{n}$ satisfy (3.1) follows from a computation similar to that in Riesz and Sz.-Nagy [18], p. 419. In addition, it is clear that

$$
\left\|P_{n}\right\| \leqq(2 \pi)^{-1}\left(\max _{z \text { on } C_{n}}\left\|(T-z)^{-1}\right\|\right) 2 \pi r_{n} \leqq r_{n} / \operatorname{dist}\left(C_{n}, \sigma(T)\right),
$$

so that (3.8) implies (3.2). Thus, by Lemma $1, P_{n} \rightarrow P$ (strongly), where $P$ is an orthogonal projection. Since $P_{n} T=T P_{n}$, then also $P T=T P$. Relation (3.10) follows from the limit relation $r_{n} \rightarrow 0$ and an estimate of $\left(T-z_{0}\right) P=-(2 \pi i)^{-1} \int_{C_{n}}\left(z-z_{0}\right)(T-z)^{-1} d z$ similar to that of (3.11).

LEMMA 3. Let $T$ be an arbitrary operator and suppose that $z_{0} \in \sigma_{p}(T)$. In addition, suppose that there exist $z_{n} \notin \sigma(T)$ such that $z_{n} \rightarrow z_{0}$ and $\left|z_{n}-z_{0}\right|\left\|\left(T-z_{n}\right)^{-1}\right\| \rightarrow 1$ as $n \rightarrow \infty$. Then $z_{0}$ is a normal eigenvalue of $T$. 
Proof. The result was given in Putnam [14] and, before this, implicitly in Stampfli [21] (cf. Stampfli's remark in [24], p. 135). A A variation appears earlier in Sz.-Nagy and Foiaș [25], p. 93. See also Hildebrandt [8], p. 234.

REMARK. Let $T$ be $G_{1}$. It is clear from Lemma 3 that if $z_{0} \in$ $\sigma_{p}(T)$ and if

$$
z_{n} \notin \sigma(T), z_{n} \longrightarrow z_{0} \text { and } \operatorname{dist}\left(z_{n}, \sigma(T)\right) /\left|z_{n}-z_{0}\right| \longrightarrow 1
$$$$
\text { as } n \longrightarrow \infty \text {, }
$$

then $z_{0}$ is a normal eigenvalue of $T$. In Lemma 2, it is assumed only that $z_{0}$ is in $\sigma(T)$ but not necessarily in $\sigma_{p}(T)$. On the other hand, the condition (3.8) for such a $z_{0}$ is clearly much stronger than (3.12). Since $T$ commutes with $P$, relation (3.10) implies that if $P \neq 0$ then necessarily $z_{0}$ is a normal eigenvalue of $T$.

If only $z_{0} \in \sigma(T)$ is assumed, it may be noted that (3.12) may hold for a completely $G_{1}$ operator, so that, in particular, $z_{0} \notin \sigma_{p}(T)$. For example, let $T$ be a completely $G_{1}$ operator as constructed in the proof of Theorem 1 , so that $T$ has the form $T=\sum \oplus\left(b_{n} A+w_{n}\right)$, where $b_{n}>0$ and $A$ is given by (2.1). If $s=\sup \operatorname{Re} \sigma(T)$, then there exists some $z_{0} \in \sigma(T)$ with $s=\operatorname{Re} z_{0}$, and hence (3.12) holds with, say, $z_{n}=z_{0}+c_{n}$, where $0<c_{n} \rightarrow 0$.

Further, note that it is possible that $T$ is $G_{1}$ with $z_{0} \in \sigma_{p}(T)$ and that there exist circles $C_{n}=\left\{z:\left|z-z_{0}\right|=r_{n}\right\}, n=1,2, \cdots$, lying in the resolvent set of $T$ and satisfying $r_{1}>r_{2}>\cdots \rightarrow 0$ and for which the projections $P_{n}$ of (3.9) are orthogonal and converge strongly to an orthogonal projection $P \neq 0$, but for which $z_{0}$ is not a normal eigenvalue of $T$. Thus, (3.10) need not hold if (3.8) is not assumed, even though the other hypotheses of Lemma 2 are retained.

A simple example is obtained by considering the construction of Stampfli ([20], [22]), with

$$
T=A \oplus N,
$$

where $A$ is given by (2.1) and $N$ is normal with spectrum $\alpha^{-}$. Here $\alpha$ is defined as in the beginning of the proof of Theorem 1 and, in particular, (2.2) holds. Clearly, for $z_{0}=0$, there exist circles $C_{n}=$ $\left\{z:|z|=r_{n}\right\}$ lying in the resolvent set of $T$ with $r_{1}>r_{2}>\cdots \rightarrow 0$. It is seen that each $P_{n}$ is an orthogonal projection. Further, if $A$ acts on the two-dimensional space $\mathfrak{S}_{0}$ then $P_{n} \rightarrow P$ (strongly), where $P$ is the projection of $\mathfrak{S}_{\mathcal{E}}$ onto $\mathscr{S}_{0}$. Although $z_{0} \in \sigma_{p}(T)$, it is clear that $z_{0}$ is not a normal eigenvalue of $T$.

The above procedure can be modified so as to yield a completely $G_{1}$ operator $T$. One need only consider the operator $T$ constructed 
in the proof of Theorem 1 above where the numbers $z_{1}, z_{2}, \cdots$, and the first sequence of disks $\left\{D_{1}, D_{2}, \cdots\right\}$, with $D_{n}=\left\{z:\left|z-z_{n}\right|<r_{n}\right\}$, are chosen so that $(0, t) \cap \bigcup_{n=1}^{\infty}\left(\left|z_{n}\right|-r_{n},\left|z_{n}\right|+r_{n}\right) \neq(0, t)$ for all $t>0$. This enables one to choose circles $C_{n}$ as in the preceding paragraph and to proceed in a manner similar to that described there.

4. THEOREM 2. Let $T$ be $G_{1}$ and suppose that $\sigma(T)$ is not a perfect set and that for each $z_{0} \in \sigma(T)$ there exists a sequence of circles $C_{n}=\left\{z:\left|z-z_{0}\right|=r_{n}\right\}, n=1,2, \cdots$, lying in the resolvent of $T$ for which $r_{1}>r_{2}>\cdots \rightarrow 0$ and (3.8) holds. Then

$T$ is normal if $\sigma(T)$ is countable,

and

$$
T=T_{1} \oplus T_{2} \text { if } \sigma(T) \text { is not countable, }
$$

where $T_{1}$ is normal with $\sigma\left(T_{1}\right)=\alpha^{-}$and $\alpha$ a countable set, and where $\sigma\left(T_{2}\right)$ is perfect and $\sigma\left(T_{2}\right) \cap \alpha=\varnothing$.

Proof. Since $\sigma(T)$ is not perfect, $\sigma(T)$ contains a nonempty (countable) set, $S_{0}$, of isolated points. Hence, as noted earlier, $T$ has a normal part $N_{0}$ corresponding to these points with $\sigma\left(N_{0}\right)=S_{0}^{-}$. In case $S_{0}=\sigma(T)$, the proof is complete. Otherwise, as will be assumed, $T=N_{0} \oplus A_{0}$, where $\sigma\left(A_{0}\right) \cap S_{0}=\varnothing$, and we let $S_{1}$ denote the (countable) set of isolated points of the first derivative, $\sigma^{\prime}(T)$, of $\sigma(T)$. If $S_{1}$ is empty the proof is over and so we can suppose that $S_{1} \neq \varnothing$. It follows from (3.10) of Lemma 2 that each point $z_{0}$ of $S_{1}$ either corresponds to a normal eigenvalue (if $P \neq 0$ ), or, if $P=0$, can simply be ignored. Thus, at the end of the second stage we have $T=$ $N_{1} \oplus A_{1}$ where $\sigma\left(N_{1}\right)=S_{0}^{-} \cup S_{1}^{-}$and, if $A_{1}$ is present, $\sigma\left(A_{1}\right) \cap\left(S_{0} \cup S_{1}\right)=$ $\varnothing$. One then repeats this process. It should be noted that for $n=0,1,2, \cdots, S_{n}=\sigma^{(n)}(T)-\sigma^{(n+1)}(T)$, where $\sigma^{(n)}(T)$ denotes the $n$th derived set of $\sigma(T) \equiv \sigma^{(0)}(T)$. If for any positive integer $n, S_{n}$ is empty, the process terminates. In addition, if $\sigma(T)=\bigcup_{n=0}^{\infty} S_{n}$, the process also terminates, and, of course, implies that $T$ is normal and that $\sigma(T)$ is countable. Otherwise, the process continues via transfinite induction as noted below.

The $\nu$ th derived set of $\sigma(T)$ can be defined, in the manner of Cantor using transfinite induction, for any ordinal $\nu$; see Kamke [9], p. 127. It follows from a transfinite induction argument ([9], pp. 132-133) that there is a least ordinal $\gamma$, where $0 \leqq$ cardinality of $\gamma \leqq \aleph_{0}$, with the property that $\sigma^{(\gamma)}(T)=\sigma^{(\alpha)}(T)$ for all ordinals $\alpha \geqq \gamma$. In particular, if $\sigma^{(r)}(T)$ is not empty then it is perfect. It follows (cf. [9], p. 133) that if $\sigma(T)$ is countable, then $\sigma^{(r)}(T)$ is empty and, 
by the process described in the preceding paragraph, (4.1) is established. If $\sigma(T)$ is not countable then $\sigma^{(r)}(T)$ is perfect and so (4.2) holds with the properties described in Theorem 2.

5. THEOREM 3. Let $T$ be $G_{1}$. Suppose that for every $\varepsilon>0$ there exists a countable covering of $\sigma(T)$ by open disks $D_{n}=\left\{z:\left|z-z_{n}\right|<r_{n}\right\}$, $n=1,2, \cdots$, with the properties that, for each $n, D_{n} \cap \sigma(T) \neq \varnothing$ and $C_{n}=\left\{z:\left|z-z_{n}\right|=r_{n}\right\}$ lies in the resolvent set of $T$, and that

$$
\sum_{n}\left(r_{n} / d_{n}-1\right)^{1 / 2}<\varepsilon, \text { where } d_{n}=\operatorname{dist}\left(C_{n}, \sigma(T)\right) \quad\left(\leqq r_{n}\right),
$$

and

$$
\sum_{n} r_{n}<\varepsilon
$$

Then $T$ is normal.

Proof. Let $\varepsilon>0$ be fixed. In view of the Heine-Borel theorem it may be suppose that the covering of Theorem 3 is finite, say $\left\{D_{1}, \cdots, D_{N}\right\}$, and that $D_{n} \not \subset D_{m}$ for $n \neq m$. For $n=1, \cdots, N$, define $P_{n}=-(2 \pi i)^{-1} \int_{C_{n}}(T-z)^{-1} d z$, where the $C_{n}$ are regarded as positively oriented, so that, by an estimate similar to that of (3.11), $\left\|P_{n}\right\| \leqq$ $r_{n} / d_{n}$. (Note that in the present case, $D_{n} \cap \sigma(T) \neq \varnothing$ but it is not assumed as in Lemma 2 that the center of $C_{n}$ is in $\sigma(T)$.) Next, if $t_{n}=r_{n} / d_{n}-1$ then $\left\|P_{n}\right\| \leqq 1+t_{n}$ (cf. (3.3)). It follows from (3.5) with $P$ and $t$ replaced by $P_{n}$ and $t_{n}$ that

$$
\left\|P_{n}-P_{n}^{*}\right\| \leqq \operatorname{const}\left(r_{n} / d_{n}-1\right)^{1 / 2} \quad(n=1, \cdots, N),
$$

provided, say, $0<\varepsilon \leqq 1 / 2$, as will be assumed. Thus, in view of (5.1).

$$
\sum_{n=1}^{N}\left\|P_{n}-P_{n}^{*}\right\| \leqq \text { const } \varepsilon
$$

Next, consider any pair or circles, say $C_{1}$ and $C_{2}$. It will be shown that if $D_{1} \cap D_{2} \neq \varnothing$ then either one circle, say $C_{2}$, can be discarded or it can be deformed into a rectifiable simple closed curve $C_{2}^{\prime}$ lying in the resolvent set of $T$ and with the properties that

$$
P_{2}=P_{C_{2}^{\prime}}=-(2 \pi i)^{-1} \int_{C_{2}^{\prime}}(T-z)^{-1} d z
$$

and

$$
\operatorname{int} C_{2}^{\prime} \subset D_{2} \text { and } D_{1} \cap \operatorname{int} C_{2}^{\prime}=\varnothing .
$$


To see this, note first that $\sigma(T) \cap\left\{z: r_{1}-d_{1}<\left|z-z_{1}\right|<r_{1}+d_{1}\right\}=$ $\varnothing$. If $D_{2} \subset\left\{z:\left|z-z_{1}\right|<r_{1}+d_{1}\right\}$, then $D_{2} \cap \sigma(T) \subset D_{1} \cap \sigma(T)$ and so $C_{2}$ can be discarded. Also, in case $D_{2} \cap\left\{z:\left|z-z_{1}\right| \leqq r_{1}-d_{1}\right\}=\varnothing$, then, since $D_{2} \not \subset D_{1}, C_{2}$ can be deformed into $C_{2}^{\prime}$ so as to satisfy both (5.5) and (5.6). The remaining possibility is that

$$
D_{2} \cap\left\{z:\left|z-z_{1}\right| \leqq r_{1}-d_{1}\right\} \neq \varnothing \quad \text { and } \quad D_{2} \not \subset\left\{z:\left|z-z_{1}\right|<r_{1}+d_{1}\right\} .
$$

It may be supposed, however, that $\left\{z:\left|z-z_{1}\right| \leqq r_{1}-d_{1}\right\} \not \subset D_{2}$ since, otherwise, $D_{1} \cap \sigma(T) \subset D_{2} \cap \sigma(T)$ and $C_{1}$ can be discarded. Consequently, $r_{2}>d_{1}$ and $d_{2}<2\left(r_{1}-d_{1}\right)$, so that $r_{2} / d_{2}>d_{1} / 2\left(r_{1}-d_{1}\right)=1 / 2\left(r_{1} / d_{1}-1\right)^{-1}$. Hence, $r_{2} / d_{2}>1 / 2 \varepsilon^{2}$, in view of, and in contradiction to (5.1) (with $\varepsilon \leqq 1 / 2)$.

Repeated applications of the above argument show that the circles $C_{1}, \cdots, C_{N}$ may be replaced by rectifiable simple closed curves, say, $\gamma_{1}, \cdots, \gamma_{M}(M \leqq N)$, where each $\gamma_{i}$ is some $C_{j}$ or some $C_{j}^{\prime}$, and where int $\gamma_{n} \cap$ int $\gamma_{m}=\varnothing$ for $m \neq n$ and $\sigma(T) \subset \bigcup_{n=1}^{M}$ int $\gamma_{n}$. It is seen from relations corresponding to (5.5) and (5.6) that $\sum_{n=1}^{M} P_{n}=I$, where $P_{n}=-(2 \pi i)^{-1} \int_{r_{n}}(T-z)^{-1} d z$, and hence that $\Sigma^{\prime} P_{n}=I$ where the prime denotes that the summation is over a subset of $\{1, \cdots, N\}$. As a result, we revert to the original notation and suppose without loss of generality, that

$$
I=\sum P_{n} \quad\left(\sum=\sum_{1}^{N}\right)
$$

It is now easy to complete the proof of Theorem 3. For,

$$
T=T I=\sum T P_{n}=\sum z_{n} P_{n}+\sum\left(T-z_{n}\right) P_{n} .
$$

But $\left\|\left(T-z_{n}\right) P_{n}\right\| \leqq r_{n}\left\|P_{n}\right\| \leqq r_{n}\left(r_{n} / d_{n}\right)<r_{n}\left(1+\varepsilon^{2}\right)$, the last inequality by (5.1). Since $\varepsilon \leqq \frac{1}{2}$, (5.2) shows that $\sum\left\|\left(T-z_{n}\right) P_{n}\right\| \geqq 2 \varepsilon$. Also, $\sum z_{n} P_{n}=\sum z_{n} P_{n}^{*}+\sum z_{n}\left(P_{n}-P_{n}^{*}\right)$ and, by (5.4), $\sum\left\|z_{n}\left(P_{n}-P_{n}^{*}\right)\right\| \leqq$ $\left(\max \left|z_{n}\right|\right)$ const $\varepsilon$. Since each $D_{n}$ contains part of $\sigma(T)$ it is clear from (5.2) that $\max \left|z_{n}\right| \leqq\|T\|+2 \varepsilon \leqq\|T\|+1$, and so, by (5.8),

$$
T=\sum_{n} P_{n}^{*}+A, \text { where }\|A\| \leqq \text { const } \varepsilon .
$$

Hence, $T^{*} T=\sum z_{n} T^{*} P_{n}^{*}+T^{*} A=\sum z_{n}\left[\bar{z}_{n} P_{n}^{*}+\left(T_{n}^{*}-\bar{z}_{n}\right) P_{n}^{*}\right]+T^{*} A$. But $\left\|T^{*} A\right\| \leqq$ const $\varepsilon$ and, as above, $\sum\left\|z_{n}\left(T_{n}^{*}-\bar{z}_{n}\right) P_{n}^{*}\right\| \leqq\left(\max \left|z_{n}\right|\right) 2 \varepsilon$, and so another application of (5.4) yields $\left\|T^{*} T\right\|-\sum\left|z_{n}\right|^{2} P_{n} \|<$ const $\varepsilon$. A similar argument yields the same inequality with $T$ and $T^{*}$ interchanged, hence $T$ is normal, and the proof is complete.

REMARKs. It is readily seen that Theorem 3 implies the assertion of Theorem 2 when $\sigma(T)$ is countable. We do not know whether the hypothesis of Theorem 2 implies that $T$ is normal even when $\sigma(T)$ 
is not countable, in which case Theorem 2 would imply Theorem 3. The hypothesis (3.8) of Theorem 2 is of course a "sparseness" condition on $\sigma(T)$ and, conceivably, is restrictive enough to imply normality of $T$. In the same vein, we do not know whether the condition (5.2) in the hypothesis of Theorem 3 is essential, although, of course, at least a boundedness restriction must be placed on the $r_{n}$ 's of (5.1). (Note that if $C_{r}$ is the circle with center at $z=0$ and radius $r$ then $r / \operatorname{dist}\left(C_{r}, \sigma(T)\right) \rightarrow 1$ as $r \rightarrow \infty$.) It is clear, of course, that (5.2) alone is not enough, since this condition amounts only to requiring that $\sigma(T)$ be of one-dimensional Hausdorff measure 0 .

It may be noted that there exist uncountable sets, corresponding to $\sigma(T)$, for which (3.8) holds. To see this, one need only modify the construction of the standard Cantor set so that the length of each removed complementary open interval is a fraction sufficiently close to 1 of the length of the (closed) interval from which it was removed.

6. Real parts of $G_{1}$ operators. If $T$ is $G_{1}$ then, as was shown in Putnam [13], p. 509,

$$
\operatorname{Re} \sigma(T) \subset \sigma(\operatorname{Re} T) .
$$

For another proof, see Berberian [1], where it is also shown that, if $\sigma(T)$ is connected,

$$
\operatorname{Re} \sigma(T)=\sigma(\operatorname{Re} T) .
$$

That (6.2) need not hold in general, however, can be deduced from the example of Stamplli of (3.13) above, simply by choosing the sequence $\left\{z_{1}, z_{2}, \cdots\right\}$ so that, for instance, $\operatorname{Re} z_{n} \neq \pm 1 / 2$ for all $n$. Then $\operatorname{Re} \sigma(T)$ consists of 0 and the real parts of the $z_{n}$ 's while $\sigma(\operatorname{Re} T)=\operatorname{Re} \sigma(T) \cup\{ \pm 1 / 2\}$. A consideration of the operator $T$ constructed in Theorem 1 , where now the disks $D_{n}$ are chosen so that $\operatorname{Re} z \neq \pm 1 / 2$ for $z \in D_{n}(n=1,2, \cdots)$, shows that (6.1) may hold properly also if $T$ is completely $G_{1}$.

It is known that (6.2) always holds for hyponormal operators; see Putnam [12], p. 46. In view of certain known results concerning the spectra of completely subnormal and completely hyponormal operators one has the following

THEOREM 4. Let $T$ have the rectangular form $T=H+i J$ and let $X$ be a compact subset of the real line. Then:

(i) $X$ is the spectrum of $H=\operatorname{Re} T$ for some completely subnormal $T$ if and only if $X$ is the closure of an open subset of the real line; 
(ii) $X$ is the spectrum of $H=\operatorname{Re} T$ for some completely hyponormal $T$ if and only if, for every open interval $I$, meas $_{1}(X \cap I)>0$ whenever $X \cap I \neq \varnothing$, where meas $_{1}$ denotes linear Lebesgue measure.

Proof of (i). First, let $X$ be the closure of an open set of real numbers, so that $X=\left(\cup I_{n}\right)^{-}$, where $I_{1}, I_{2}, \cdots$ is a countable set of pairwise disjoint open intervals. Since the unilateral shift $V$ is subnormal and $\sigma(V)$ is the closed unit disk (see, e.g., Halmos [7]), one need only put $T=\sum \oplus\left(a_{n} V+b_{n}\right)$ where $a_{n}, b_{n}$ are real, $a_{n}>0$, and $I_{n}=\left(-a_{n}+b_{n}, a_{n}+b_{n}\right)$. Clearly, $X \subset \sigma(T)$, while the reverse inclusion follows from the fact that each term $a_{n} V+b_{n}$ is $G_{1}$.

Conversely, suppose that $H=\operatorname{Re} T$ where $T$ is completely subnormal and let $X=(\operatorname{int} \sigma(H))^{-}$. It will be shown that $X=\sigma(H)$. If $X \neq \sigma(H)$, then there exists some $c \in \sigma(H)-X$ and an open interval $I_{c}$ containing $c$ such that $\sigma(H) \cap I_{c}$ has no interior. In view of (6.2), there exists an open disk $D$ intersecting $\sigma(T)$ for which $Y=\sigma(T) \cap D^{-}$ is nowhere dense and has a connected complement. Hence $C(Y)=$ $P(Y)$, by Lavrentiev's theorem (cf. Gamelin [5], y. 48), and hence $T$ has a normal part with spectrum $Y$; see Clancey and Putnam [4]. Thus, $T$ is not completely subnormal, a contradiction.

Proof of (ii). First, suppose that $X \cap I$ has positive linear measure whenever $I$ is an open interval and $X \cap I$ is not empty. Let $T=H+i J$ on $\mathfrak{F}=L^{2}(X)$, where $(H x)(t)=t x(t)$ and $(J x)(t)=$ $-(i \pi)^{-1} \int_{X}(s-t)^{-1} x(s) d s$, the integral regarded as a Cauchy principal value. Then $T$ is completely hyponormal, $\sigma(T)=X \times[-1,1]$, and $\operatorname{Re} \sigma(T)=X$; cf. Clancey and Putnam [3], p. 452.

Next, suppose that $H=\operatorname{Re} T$ where $T$ is completely hyponormal. Then $\sigma(T) \cap D$ has positive planar measure whenever $D$ is an open disk for which $\sigma(T) \cap D$ is not empty; see Putnam [15], p. 324. Since $T$ satisfies (6.2), it is clear that $\sigma(H) \cap I$ has positive linear measure whenever $I$ is an open interval for which $\sigma(H) \cap I$ is not empty. This completes the proof of Theorem 4 .

As was noted in $\S 1$, a necessary and sufficient condition on a compact set of the plane in order that it be the spectrum of a completely $G_{1}$ operator is not known. Also, we do not have an analogue of Theorem 4. However, it is possible to prove the following

THEOREM 5. In order that a compact set $X$ of the real line be the spectrum of the real part of a completely $G_{1}$ operator $T$ it is necessary that $X$ be uncountable (equivalently, that $X$ contain a perfect set). 
Proof. In view of (6.1) it is clear that if $T$ is any $G_{1}$ operator and if $X=\sigma(\operatorname{Re} T)$ then $\sigma(T)$ is contained in the set consisting of all lines $\{z: \operatorname{Re} z=c\}$ where $c \in X$. Further, since $T$ of the theorem is completely $G_{1}$, then $\{z: \operatorname{Re} z=c\} \cap \sigma(T)$ is empty whenever $c$ is an isolated point of $X$, as can be seen from (6.1) and Stampfli's result ([22], [23]) mentioned in $\S 1$. Consequently, $\sigma(T)$ is contained in the union of lines $\{z: \operatorname{Re} z=c\}$ where $c \in X^{\prime}$, the first derived set of $X$. As above, no point of $\sigma(T)$ can lie on $\{z: \operatorname{Re} z=c\}$ if $z$ is an isolated point of $X^{\prime}$, that is if $c \notin X^{\prime \prime}$. It follows as in the proof of Theorem 2 that if $\gamma$ is the least ordinal (necessarily of finite or denumerable cardinality) with the property that $X^{(r)}=X^{(r+1)}$ then necessarily $\sigma(T)$ is contained in the union of lines $\{z: \operatorname{Re} z=c\}$ with $c \in X^{(r)}$. Consequently, $X^{(r)} \neq \varnothing$, hence is perfect, and the proof of Theorem 5 is complete.

REMARKs. In Theorem 5 it is possible that $X$ contains some isolated points. One need only consider the example mentioned at the beginning of this section illustrating that (6.1) may be a proper inclusion with $T$ completely $G_{1}$. We do not know whether the condition of Theorem 5 on $X$ is also sufficient, that is, whether any uncountable compact set of the real line must be the spectrum of the real part of some completely $G_{1}$ operator.

\section{REFERENCES}

1. S. K. Berberian, Conditions on an operator implying $\operatorname{Re} \sigma(T)=\sigma(\operatorname{Re} T)$, Trans. Amer. Math. Soc., 154 (1971), 267-272.

2. L. Carleson, Selected Problems on Exceptional Sets, D. van Nostrand Co., Inc., Princeton, 1967.

3. K. F. Clancey and C. R. Putnam, The spectra of hyponormal integral operators, Comm. Math. Helv., 46 (1971), 451-456.

4. - The local spectral behavior of completely subnormal operators, Trans. Amer. Math. Soc., 163 (1972), 239-244.

5. T. W. Gamelin, Uniform Algebras, Prentice-Hall, Inc., Englewood Cliffs, N. J., 1969.

6. J. Garnett, Analytic Capacity and Measure, Lecture notes in mathematics, 297, Springer, Berlin, 1972.

7. P. R. Halmos, A Hilbert Space Problem Book, D. van Nostrand Co., Inc., Princeton, 1967.

8. S. Hildebrandt, Über den numerischen Wertebereich eines Operators, Math. Ann., 163 (1966), 230-247.

9. E. Kamke, Theory of Sets, Dover Publications, New York, 1950.

10. G. R. Luecke, Operators satisfying condition $\left(G_{1}\right)$ locally, Pacific J. Math., 40 (1972), 629-637.

11. Topological properties of paranormal operators on Hilber space, Trans. Amer. Math. Soc., 172 (1972), 35-43.

12. C. R. Putnam, Commutation Properties of Hilbert Space Operators and Related Topics, Ergebnisse der Math., 36, Springer, Berlin, 1967.

13. - The spectra of operators having resolvents of first-order growth, Trans. 
Amer. Math. Soc., 133 (1968), 505-510.

14. — Eigenvalues and boundary spectra, Illinois J. Math., 12 (1968), 278-282.

15. — An inequality for the area of hyponormal spectra, Math. Zeits., 116 (1970), 323-330.

16. - Almost normal operators, their spectra and invariant subspaces, Bull. Amer. Math. Soc., 79 (1973), 615-624.

17. The role of zero sets in the spectra of hyponormal operators, Proc. Amer. Math. Soc., 43 (1974), 137-140.

18. F. Riesz and B. Sz.-Nagy, Functional Analysis, Frederick Ungar Pub. Co., New York, 1955.

19. C. A. Rogers, Hausdorff Measures, Cambridge Univ. Press, 1970.

20. J. G. Stampfli, Hyponormal operators and spectral density, Trans. Amer. Math. Soc., 117 (1965), 469-476.

21. - Analytic extensions and spectral localization, J. Math. Mech., 16 (1966), 287-296.

22. ——, A local spectral theory for operators, J. Functional Anal., 4 (1969), 1-10.

23. - A local spectral theory for operators, II, Bull. Amer. Math. Soc., 75 (1969), 803-806.

24. - A local spectral theory for operators, III: resolvents, spectral sets and similarity, Trans. Amer. Math. Soc., 168 (1972), 133-151.

25. B. Sz.-Nagy and C. Foiaș, Une relation parmi les vecteurs propersd'un opérateur de l'espace de Hilbert et de l'opérateur adjoint, Acta Sci. Math. (Szeged), 20 (1959), 91-96.

26. A. Wintner, Zur Theorie der beschrankten Bilinearformen, Math. Zeits., 30 (1929), 228-282.

Received September 23, 1977. This work was supported by a National Science Foundation research grant.

PURDUE UNIVERSITY

WEST LAFAYETTE, IN 47907 


\section{PACIFIC JOURNAL OF MATHEMATICS}

\section{EDITORS}

DONALD BABBITT (Managing Editor)

University of California

Los Angeles, CA 90024

HUGO RossI

University of Utah

Salt Lake City, UT 84112

C. C. Moore and ANDrew OGG

University of California

Berkeley, CA 94720
J. DUGUNDJI

Department of Mathematics University of Southern California Los Angeles, CA 90007

R. FINN and J. MILGRAM

Stanford University

Stanford, CA 94305

\section{ASSOCIATE EDITORS}
E. F. BeCKENBACH
B. H. NeumanN
F. WOLF
K. YOSHIDA

\section{SUPPORTING INSTITUTIONS}

UNIVERSITY OF BRITISH COLUMBIA CALIFORNIA INSTITUTE OF TECHNOLOGY UNIVERSITY OF CALIFORNIA MONTANA STATE UNIVERSITY UNIVERSITY OF NEVADA, RENO NEW MEXICO STATE UNIVERSITY OREGON STATE UNIVERSITY UNIVERSITY OF OREGON

\author{
UNIVERSITY OF SOUTHERN CALIFORNIA \\ STANFORD UNIVERSITY \\ UNIVERSITY OF HAWAII \\ UNIVERSITY OF TOKYO \\ UNIVERSITY OF UTAH \\ WASHINGTON STATE UNIVERSITY \\ UNIVERSITY OF · WASHINGTON
}

The Supporting Institutions listed above contribute to the cost of publication of this Journal, but they are not owners or publishers and have no responsibility for its content or policies.

Mathematical papers intended for publication in the Pacific Journal of Mathematics should be in typed form or offset-reproduced, (not dittoed), double spaced with large margins. Please do not use built up fractions in the text of the manuscript. However, you may use them in the displayed equations. Underline Greek letters in red, German in green, and script in blue. The first paragraph or two must be capable of being used separately as a synopsis of the entire paper. Please propose a heading for the odd numbered pages of less than 35 characters. Manuscripts, in triplicate, may be sent to any one of the editors. Please classify according to the scheme of Math. Reviews, Index to Vol. 39. Supply name and address of author to whom proofs should be sent. All other communications should be addressed to the managing editor, or Elaine Barth, University of California, Los Angeles, California, 90024.

50 reprints to each author are provided free for each article, only if page charges have been substantially paid. Additional copies may be obtained at cost in multiples of 50 .

The Pacific Journal of Mathematics is issued monthly as of January 1966. Regular subscription rate: $\$ 84.00$ a year (6 Vols., 12 issues). Special rate: $\$ 42.00$ a year to individual members of supporting institutions.

Subscriptions, orders for numbers issued in the last three calendar years, and changes of address should be sent to Pacific Journal of Mathematics, P.O. Box 969, Carmel Valley, CA 93924, U.S.A. Older back numbers obtainable from Kraus Periodicals Co., Route 100, Millwood, NY 10546.

PUBLISHED BY PACIFIC JOURNAL OF MATHEMATICS, A NON-PROFIT CORPORATION

Printed at Kokusai Bunken Insatsusha (International Academic Printing Co., Ltd.). 8-8, 3-chome, Takadanobaba, Shinjuku-ku, Tokyo 160, Japan.

Copyright (C) 1979 by Pacific Journal of Mathematics Manufactured and first issued in Japan 


\section{Pacific Journal of Mathematics}

\section{Vol. 84, No. $2 \quad$ June, 1979}

Somesh Chandra Bagchi and Alladi Sitaram, Spherical mean periodic

functions on semisimple Lie groups ........................ 241

Billy Joe Ball, Quasicompactifications and shape theory............. 251

Maureen A. Bardwell, The o-primitive components of a regular ordered permutation group ................................ 261

Peter W. Bates and James R. Ward, Periodic solutions of higher order

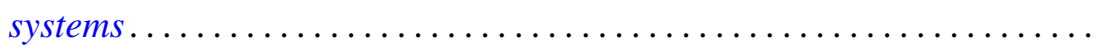

Jeroen Bruijning, A characterization of dimension of topological spaces by totally bounded pseudometrics......................... 283

Thomas Farmer, On the reduction of certain degenerate principal series

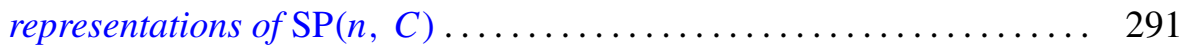

Richard P. Jerrard and Mark D. Meyerson, Homotopy with m-functions . . . . 305

James Edgar Keesling and Sibe Mardesic, A shape fibration with fibers of

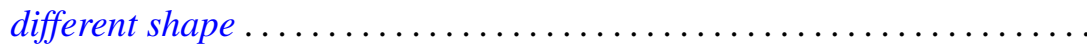

Guy Loupias, Cohomology over Banach crossed products. Application to bounded derivations and crossed homomorphisms ...............

Rainer Löwen, Symmetric planes ........................ 367

Alan L. T. Paterson, Amenable groups for which every topological left

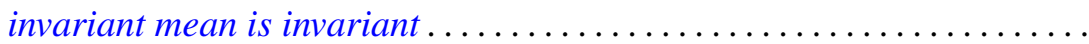

Jack Ray Porter and R. Grant Woods, Ultra-Hausdorff H-closed extensions

Calvin R. Putnam, Operators satisfying a $G_{1}$ condition .

Melvin Gordon Rothenberg and Jonathan David Sondow, Nonlinear smooth representations of compact Lie groups..............

Werner Rupp, Riesz-presentation of additive and $\sigma$-additive set-valued measures.

A. M. Russell, A commutative Banach algebra of functions of generalized variation

Judith D. Sally, Superregular sequences

Patrick Shanahan, On the signature of Grassmannians . . 